\title{
Towards accelerated thermoelectric materials and process discovery
}

Jose Recatala-Gomez ${ }^{\star} \#$, Ady Suwardi ${ }^{\star \#, ~ I r i s ~ N a n d h a k u m a r ~}{ }^{\perp *}$, Anas Abutaha ${ }^{\star *}$, Kedar Hippalgaonkar ${ }^{\sharp * *}$

$\S$ School of Material Science and Engineering, Nanyang Technological University, Singapore 639798.

*Institute of Materials Research and Engineering, \#08-03, 2 Fusionopolis Way, Agency for Science, Technology and Research, Singapore 138634.

${ }^{\perp}$ Department of Chemistry, University of Southampton, University Road, Highfield, Southampton SO17 1BJ, United Kingdom.

\# equal contribution

* corresponding authors: kedar@ntu.edu.sg; iris@ soton.ac.uk; anasabutaha@gmail.com

\begin{tabular}{|c|c|c|c|c|c|c|c|c|}
\hline \multicolumn{9}{|c|}{ Supplementary Table S1 } \\
\hline Material & $\begin{array}{l}\text { Publication } \\
\text { Year }\end{array}$ & $\begin{array}{l}\mathrm{ZT} \\
\text { value }\end{array}$ & $\begin{array}{l}\text { Temperature } \\
\text { of Peak ZT }(K)\end{array}$ & $\begin{array}{l}\text { Scientific } \\
\text { Rating }\end{array}$ & $\begin{array}{l}\text { Technological } \\
\text { Rating }\end{array}$ & System & Color & Reference \\
\hline $\begin{array}{l}\mathrm{Bi0} 0.5 \mathrm{Sb} 1.5 \mathrm{~T} \\
\mathrm{e} 3\end{array}$ & 2015 & 1.86 & 320 & 2 & 4 & A & Red & $\begin{array}{l}\text { https://science.sciencemag.org/content/348/6230/ } \\
109\end{array}$ \\
\hline $\begin{array}{l}\text { Nanobulk } \\
\text { BST }\end{array}$ & 2008 & 1.40 & 375 & 3 & 4 & $\mathrm{~A}$ & Red & $\begin{array}{l}\mathrm{https}: / / \text { science.sciencemag.org/content/320/5876/ } \\
634\end{array}$ \\
\hline $\mathrm{SiGe}$ & 1964 & 0.90 & 1100 & 2 & 1 & B & Blue & $\begin{array}{l}\text { https://journals.aps.org/pr/abstract/10.1103/Phys } \\
\text { Rev.134.A1058 }\end{array}$ \\
\hline $\mathrm{SiGe}$ & 2008 & 0.95 & 1100 & 4 & 4 & $\mathrm{~B}$ & Blue & https://pubs.acs.org/doi/10.1021/n18026795 \\
\hline SiGe & 2012 & 1.30 & 1173 & 3.5 & 3 & B & Blue & https://pubs.acs.org/doi/10.1021/nl3003045 \\
\hline SiGe & 2014 & 1.84 & 1073 & 4 & 3.5 & $\mathrm{~B}$ & Blue & $\begin{array}{l}\text { https://pubs.rsc.org/en/content/articlelanding/201 } \\
\text { 4/ta/c3ta14259k\#!divAbstract }\end{array}$ \\
\hline $\mathrm{Mg} 3 \mathrm{Bi} 2$ & 2019 & 0.90 & 350 & 5.25 & 3 & $\mathrm{C}$ & Green & $\begin{array}{l}\text { https://science.sciencemag.org/content/365/6452/ } \\
\text { 495.abstract }\end{array}$ \\
\hline
\end{tabular}




\begin{tabular}{|c|c|c|c|c|c|c|c|c|}
\hline $\begin{array}{l}\text { Te and } \mathrm{Bi} \\
\text { doped } \\
\mathrm{Mg} 3 \mathrm{Sb} 2\end{array}$ & 2017 & 1.65 & 725 & 4.75 & 1.75 & $\mathrm{C}$ & Green & $\begin{array}{l}\text { https://www.nature.com/articles/ncomms13901.p } \\
\underline{\text { df }}\end{array}$ \\
\hline $\begin{array}{l}\mathrm{MNC}-\mathrm{Ni} / \mathrm{Fe} \\
\text { nanoparticle } \\
\mathrm{S}\end{array}$ & 2017 & 1.75 & 900 & 5.1 & 2.8 & $\mathrm{D}$ & Salmon & https://www.nature.com/articles/nature23667 \\
\hline $\mathrm{PbSe}$ & 2019 & 1.70 & 300 & 4.8 & 2.9 & $\mathrm{E}$ & Grey & $\begin{array}{l}\text { https://www.nature.com/articles/s41563-019- } \\
\text { 0499-9 }\end{array}$ \\
\hline $\begin{array}{l}\mathrm{Pb} 0.98 \mathrm{Na} 0.0 \\
2 \mathrm{Se}+3 \% \\
\mathrm{CdS}\end{array}$ & 2013 & 1.60 & 973 & 4.5 & 3.2 & $\mathrm{E}$ & Grey & https://pubs.acs.org/doi/10.1021/ja403134b \\
\hline $\begin{array}{l}\text { Br doped } \\
\mathrm{PbSe}\end{array}$ & 2012 & 1.00 & 800 & 2.5 & 2.5 & $\mathrm{E}$ & Grey & https://www.pnas.org/content/109/25/9705 \\
\hline $\begin{array}{l}\mathrm{Pb} 0.7 \mathrm{Sn} 0.3 \mathrm{~T} \\
\mathrm{e} 0.9 \mathrm{Se} 0.1\end{array}$ & 2019 & 0.50 & 300 & 0.5 & 5 & $E$ & Grey & $\begin{array}{l}\text { https://pubs.rsc.org/en/content/articlelanding/201 } \\
\text { 9/me/c8me00073e\#!divAbstract }\end{array}$ \\
\hline PbTe-SnTe & 2019 & 0.50 & 300 & 0.7 & 5 & $\mathrm{E}$ & Grey & $\begin{array}{l}\text { https://pubs.acs.org/doi/abs/10.1021/acscombsci. } \\
\text { 9b00145 }\end{array}$ \\
\hline $\mathrm{GeTe}$ & 2009 & 1.80 & 773 & 4 & 3 & $\mathrm{~F}$ & Plum & $\begin{array}{l}\text { https://link.springer.com/article/10.1007/s11664- } \\
\text { 009-1012-z }\end{array}$ \\
\hline GeTe & 2019 & 2.10 & 723 & 4.7 & 2.8 & $\mathrm{~F}$ & Plum & $\begin{array}{l}\text { https://www.sciencedirect.com/science/article/ab } \\
\text { s/pii/S2542435119304209 }\end{array}$ \\
\hline GeTe & 2018 & 2.40 & 600 & 5 & 2.25 & $\mathrm{~F}$ & Plum & $\begin{array}{l}\text { https://www.cell.com/joule/pdf/S2542- } \\
\text { 4351(18)30085-0.pdf }\end{array}$ \\
\hline GeTe & 2019 & 2.10 & 800 & 4.9 & 3.25 & $\mathrm{~F}$ & Plum & $\begin{array}{l}\text { https://pubs.rsc.org/en/Content/ArticleLanding/2 } \\
\text { 019/TA/C9TA10963C\#!divAbstract }\end{array}$ \\
\hline $\mathrm{GeTe}$ & 2019 & 1.90 & 723 & 4.75 & 2.6 & $\mathrm{~F}$ & Plum & https://pubs.acs.org/doi/10.1021/acsaem.9b01585 \\
\hline $\begin{array}{l}1 \% \text { mol In } \\
\text { doped } \\
\text { Cu2Se }\end{array}$ & 2017 & 2.60 & 850 & 3.5 & 3.5 & $\mathrm{G}$ & Magenta & $\begin{array}{l}\text { https://pubs.rsc.org/en/content/articlelanding/201 } \\
\text { 7/ee/c7ee01193h\#!divAbstract }\end{array}$ \\
\hline $\begin{array}{l}\text { Dispersed } \\
\text { MWCNT in } \\
\text { Cu2Se }\end{array}$ & 2017 & 2.40 & 1000 & 3 & 2.5 & $\mathrm{G}$ & Magenta & $\begin{array}{l}\text { https://pubs.rsc.org/en/content/articlelanding/201 } \\
\text { 7/ee/c7ee01737e\#!divAbstract }\end{array}$ \\
\hline Cu2Se1-xIx & 2013 & 2.29 & 400 & 3.5 & 2.7 & G & Magenta & $\begin{array}{l}\text { https://onlinelibrary.wiley.com/doi/abs/10.1002/a } \\
\text { dma.201302660 }\end{array}$ \\
\hline $\begin{array}{l}\mathrm{Ba} 0.08 \mathrm{La} 0.0 \\
5 \mathrm{Yb} 0.04 \mathrm{Co} 4 \\
\mathrm{Sb} 12\end{array}$ & 2011 & 1.70 & 850 & 3.5 & 2.5 & $\mathrm{H}$ & Pink & https://pubs.acs.org/doi/pdf/10.1021/ja111199y \\
\hline
\end{tabular}




\begin{tabular}{|c|c|c|c|c|c|c|c|c|}
\hline $\begin{array}{l}\text { LaFeCoSb1 } \\
2\end{array}$ & 1996 & 0.90 & 800 & 3.25 & 3 & $\mathrm{H}$ & Pink & $\begin{array}{l}\text { https://science.sciencemag.org/content/sci/272/52 } \\
\text { 66/1325.full.pdf }\end{array}$ \\
\hline $\begin{array}{l}\mathrm{Se} \text { and } \mathrm{Na} \\
\text { doped } \mathrm{SnS}\end{array}$ & 2019 & 1.60 & 873 & 4.7 & 0.7 & I & Gold & $\begin{array}{l}\text { https://science.sciencemag.org/content/365/6460/ } \\
1418\end{array}$ \\
\hline $\begin{array}{l}\text { Sn0.5Mn0.2 } \\
\text { Pb0.3Te }\end{array}$ & 2019 & 1.50 & 900 & 5 & 3.15 & I & Gold & $\begin{array}{l}\text { https://onlinelibrary.wiley.com/doi/10.1002/inf2. } \\
12044\end{array}$ \\
\hline $\begin{array}{l}\text { Br doped } \\
\text { SnSe }\end{array}$ & 2018 & 2.80 & 773 & 5 & 0.5 & I & Gold & $\begin{array}{l}\text { https://science.sciencemag.org/content/360/6390/ } \\
\text { 778 }\end{array}$ \\
\hline $\begin{array}{l}\text { Tl doped } \\
\text { PbTe }\end{array}$ & 2008 & 1.50 & 773 & 4.25 & 2.5 & $\mathrm{~J}$ & Teal & $\begin{array}{l}\text { https://science.sciencemag.org/content/321/5888/ } \\
554\end{array}$ \\
\hline $\begin{array}{l}\text { SrTe doped } \\
\text { PbTe }\end{array}$ & 2012 & 2.20 & 915 & 4.75 & 3.5 & $\mathrm{~J}$ & Teal & https://www.nature.com/articles/nature11439 \\
\hline $\begin{array}{l}\text { Cubic } \\
\text { AgPbmSbTe } \\
2+\mathrm{m}\end{array}$ & 2004 & 2.20 & 800 & 3.75 & 2 & $\mathrm{~J}$ & Teal & $\begin{array}{l}\text { https://science.sciencemag.org/content/303/5659/ } \\
\underline{818}\end{array}$ \\
\hline $\begin{array}{l}\text { Na doped } \\
\text { PbTe }\end{array}$ & 2011 & 1.40 & 750 & 4 & 1.75 & $\mathbf{J}$ & Teal & $\begin{array}{l}\text { https://pubs.rsc.org/en/content/articlelanding/201 } \\
\text { 1/ee/c0ee00456a\#!divAbstract }\end{array}$ \\
\hline $\begin{array}{l}\mathrm{HH} \\
\mathrm{ZrHfTiNiSn} \\
\mathrm{Sb}\end{array}$ & 2005 & 1.50 & 700 & 2 & 2 & $\mathrm{~K}$ & Olive & $\begin{array}{l}\text { https://aip.scitation.org/doi/pdf/10.1063/1.18680 } \\
\text { 63?class=pdf }\end{array}$ \\
\hline $\begin{array}{l}\text { ZrNiSn0.99 } \\
\text { Sb0.01 }\end{array}$ & 2014 & 0.80 & 875 & 1 & 3 & $\mathrm{~K}$ & Olive & https://www.nature.com/articles/srep06888.pdf \\
\hline $\begin{array}{l}\text { Hf } 0.65 \mathrm{Zr} \\
0.35 \mathrm{Ni} 1-\mathrm{z} \\
\mathrm{Pt} \text { z Sn } 0.98 \\
\text { Sb } 0.02\end{array}$ & 2013 & 0.75 & 875 & 2.15 & 3 & $\mathrm{~K}$ & Olive & $\begin{array}{l}\text { https://onlinelibrary.wiley.com/doi/full/10.1002/a } \\
\text { dfm.201300663 }\end{array}$ \\
\hline $\begin{array}{l}\text { Antifluorite } \\
\mathrm{Mg} 2 \mathrm{Si} 0.6 \mathrm{Sn} \\
0.4\end{array}$ & 2006 & 1.10 & 867 & 3 & 1 & $\mathrm{~L}$ & Indigo & $\begin{array}{l}\text { https://journals.aps.org/prb/pdf/10.1103/PhysRev } \\
\text { B.74.045207 }\end{array}$ \\
\hline $\begin{array}{l}\text { Quasi 1D } \\
\text { In4Se3 }\end{array}$ & 2009 & 1.47 & 705 & 5 & 0.75 & $\mathrm{M}$ & Crimson & https://www.nature.com/articles/nature08088.pdf \\
\hline $\begin{array}{l}\mathrm{Bi0} 0.925 \mathrm{Ca} 0 . \\
075 \mathrm{CuSeO}\end{array}$ & 2013 & 0.90 & 923 & 1 & 1.5 & $\mathrm{~N}$ & Tan & https://www.nature.com/articles/am201315.pdf \\
\hline $\begin{array}{l}\text { BiCuSe0.94 } \\
\text { Te0.06O }\end{array}$ & 2013 & 0.71 & 873 & 2 & 1.75 & $\mathrm{~N}$ & Tan & $\begin{array}{l}\text { https://pubs.rsc.org/en/content/articlelanding/201 } \\
\text { 3/CC/c3cc44578j\#!divAbstract }\end{array}$ \\
\hline $\begin{array}{l}\text { Ba doped } \\
\mathrm{BiCuSeO}\end{array}$ & 2012 & 1.10 & 923 & 1 & 3.5 & $\mathrm{~N}$ & Tan & $\begin{array}{l}\text { https://pubs.rsc.org/en/content/articlelanding/201 } \\
\text { 2/ee/c2ee22622g\#!divAbstract }\end{array}$ \\
\hline
\end{tabular}




\begin{tabular}{|c|c|c|c|c|c|c|c|c|}
\hline $\begin{array}{l}\mathrm{Bi} 0.85 \mathrm{Sr} 0.1 \\
5 \mathrm{CuSeO}\end{array}$ & 2010 & 0.76 & 873 & 3 & 2 & $\mathrm{~N}$ & Tan & $\begin{array}{l}\text { https://aip.scitation.org/doi/pdf/10.1063/1.34850 } \\
\text { 50?class=pdf }\end{array}$ \\
\hline $\begin{array}{l}\mathrm{Pb} 0.975 \mathrm{Na} 0 \\
025 \mathrm{~S}+3 \% \\
\mathrm{CdS}\end{array}$ & 2012 & 1.30 & 923 & 3.25 & 2.8 & $\mathrm{O}$ & Orange & https://pubs.acs.org/doi/10.1021/ja306527n \\
\hline $\begin{array}{l}\mathrm{PbS} 1-\mathrm{xClx} \\
(\mathrm{x} \leq 0.008)\end{array}$ & 2012 & 0.70 & 850 & 2 & 2.9 & $\mathrm{O}$ & Orange & $\begin{array}{l}\text { https://onlinelibrary.wiley.com/doi/abs/10.1002/a } \\
\text { enm.201200683 }\end{array}$ \\
\hline $\mathrm{Cu} 1.97 \mathrm{~S}$ & 2014 & 1.70 & 1000 & 2.5 & 3 & $\mathrm{P}$ & Purple & $\begin{array}{l}\text { https://onlinelibrary.wiley.com/doi/epdf/10.1002/ } \\
\text { adma.201400515 }\end{array}$ \\
\hline $\mathrm{Cu} 1.8 \mathrm{~S}$ & 2011 & 0.50 & 673 & 2 & 2.5 & $\mathrm{P}$ & Purple & $\begin{array}{l}\text { https://pubs.rsc.org/en/content/articlelanding/201 } \\
\text { 1/CC/c1cc16368j\#!divAbstract }\end{array}$ \\
\hline
\end{tabular}

